$62^{\text {ème }}$ Congrès de la SFCO, 01004 (2014)

DOI: $10.1051 /$ sfco/20146201004

(C) Owned by the authors, published by EDP Sciences, 2014

\title{
CONFÉRENCE
}

\section{Gestion des édentements étroits en secteurs esthétiques.}

\author{
Dr Philippe Russe / Pratique privée, Reims, FRANCE
}

russe-phil@orange.fr

Face à l'absence d'une ou de plusieurs dents en zone esthétique, le praticien doit suivre une démarche diagnostique générale, quel que soit le type d'édentement :

1 Évaluer les facteurs de risque médicaux et comportementaux,

2 Observer la ligne du sourire et la typologie parodontale,

3 Analyser l'esthétique existante,

4 Etudier le volume osseux implantable.

Le traitement des édentements unitaires en secteur esthétique est particulièrement délicat car le résultat prothétique sera directement comparé aux dents adjacentes et toute dissymétrie immédiatement perceptible, même pour un non-initié.

L'obtention d'un score d'évaluation esthétique des tissus mous élevé (PES: pink esthetic score de Furhauser 2005) est souvent le résultat de techniques de reconstructions osseuses et/ou gingivales.

Les édentements unitaires étroits, petites incisives latérales maxillaires et incisives mandibulaires, posent des problèmes spécifiques et sont avantageusement traités avec des matériels adaptés comme les implants de petit diamètre.

Les résultats très positifs de deux études successives sur des implants de $3 \mathrm{~mm}$ de diamètre, portant au total sur 216 implants seront présentés.
Des enseignements, tirés de l'expérience de ces 2 séries, portant sur chaque étape du traitement seront exposés :

- L'évaluation pré-opératoire du site implantaire grâce à l'imagerie tomodensitométrique à faisceau conique.

- La reconstruction osseuse éventuelle avec des autogreffes avec une attention toute particulière portée à la zone du soutien papillaire.

- La pose d'implant sous contrôle radiographique grâce à de nouveaux générateurs.

- Une gestion des tissus mous, soit lors de la pose de l'implant, soit lors de la réouverture, faisant appel à différentes techniques dérivées du lambeau enveloppe de Peter Raetzke (1985) et du split-finger de Carl Misch (2004).

- Une étape incontournable de prothèse de temporisation permettant de modeler le profil d'émergence dans le respect du principe du "One abutment, one time » proposé par les frères Salama (2008).

- Une prothèse céramique sur base de métal ou de céramique pressée en fonction de la situation clinique.

La présentation de cas traités et documentés sur une période de 10 à 20 ans permet de poser le problème de la croissance continue des maxillaires dont les facteurs de risque seront décrits.

This is an Open Access article distributed under the terms of the Creative Commons Attribution License 4.0, which permits unrestricted use, distribution, and reproduction in any medium, provided the original work is properly cited. 